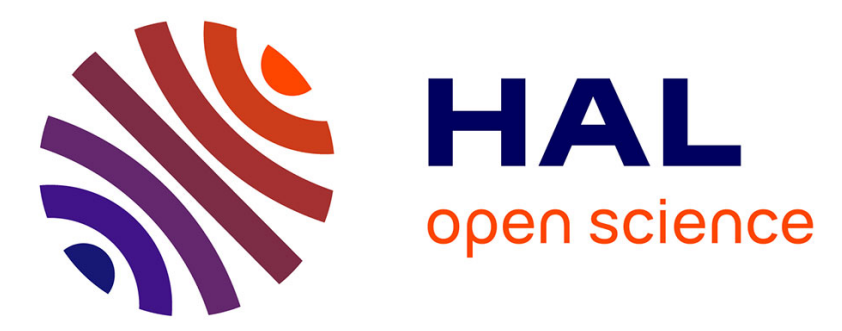

\title{
Numerical simulation of two-dimensional multiple scattering of sound by a large number of circular cylinders
}

Adrien Rohfritsch, Jean-Marc Conoir, Régis Marchiano, Tony Valier-Brasier

\section{- To cite this version:}

Adrien Rohfritsch, Jean-Marc Conoir, Régis Marchiano, Tony Valier-Brasier. Numerical simulation of two-dimensional multiple scattering of sound by a large number of circular cylinders. Journal of the Acoustical Society of America, 2019, 145 (6), pp.3320-3329. 10.1121/1.5110310 . hal-02196752v2

\section{HAL Id: hal-02196752 \\ https://hal.science/hal-02196752v2}

Submitted on 28 Jul 2020 (v2), last revised 29 Aug 2021 (v3)

HAL is a multi-disciplinary open access archive for the deposit and dissemination of scientific research documents, whether they are published or not. The documents may come from teaching and research institutions in France or abroad, or from public or private research centers.
L'archive ouverte pluridisciplinaire HAL, est destinée au dépôt et à la diffusion de documents scientifiques de niveau recherche, publiés ou non, émanant des établissements d'enseignement et de recherche français ou étrangers, des laboratoires publics ou privés. 


\title{
Numerical simulation of two-dimensional multiple scattering of sound by a large number of circular cylinders
}

\author{
Adrien Rohfritsch, ${ }^{1}$ Jean-Marc Conoir, ${ }^{1}$ Régis Marchiano, ${ }^{1}$ and Tony Valier-Brasier ${ }^{1}$ \\ Sorbonne Université, CNRS, Institut Jean Le Rond d'Alembert, UMR 7190, 4 Place Jussieu, Paris, \\ F-75005, France ${ }^{\mathrm{a})}$
}

The purpose of this article is to present an innovative resolution method for investigating problems of sound scattering by infinite cylinders immersed in a fluid medium. The study is based on the analytical solution of multiple scattering, where incident and scattered waves are expressed in cylindrical harmonics. This modeling leads to dense linear systems, which are made sparse by introducing a cutoff radius around each particle. This cutoff radius is deeply studied and quantified. Numerical resolution is performed using parallel computing methods designed to solve very large sparse linear systems. Comparisons with direct calculations, made with another numerical software, and with homogenization technics follow and show good agreement with the implemented method. The last part is dedicated to a comparison between the propagation of waves in a circular cluster made of a random distribution of cylinders and the propagation in the corresponding homogenized cluster where the multiple scattering formalism is combined with a statistical analysis to provide an effective medium.

(C)2020 Acoustical Society of America. [http://dx.doi.org(DOI number)]

$[\mathrm{XYZ}]$

Pages: $1-12$

\section{INTRODUCTION}

Multiple scattering of waves is a general problem which occurs in many fields of physics. Both the acoustical and electromagnetical physics communities have problems of great interest that deal with multiple scattering, such as the characterization of effective parameters of heterogeneous media or phononic crystals, for example.

Here, we present a study of scattering by infinite parallel cylinders. The model used in this paper to describe the wave propagation through a cluster of cylinders was first presented by F. Zàviška ${ }^{1}$. It is an analytical model which leads to writing the solution in an implicit way through a linear system of great size, which increases with frequency and the number of cylinders. In view of the difficulty to resolve this dense linear system, two strategies have emerged.

The first is based on statistical tools applied to the expression of the total acoustical field, leading to the calculation of statistical quantities, for instance self-energy or effective wave numbers. As a partial overview of notable papers based on the multiple scattering model, one can note for instance Bose et $a l^{3}{ }^{3}$ for the study of wave propagation in fiber-reinforced composites. This study is based on the quasi-cristalline approximation, introduced by $\mathrm{Lax}^{4}$, that gives exact results for crystal lattices. Later on, Varadan et al. ${ }^{5}$ generalized this case to arbitrary shaped scatterers. Le Bas et al. ${ }^{6}$ have introduced the S-matrix to study the resonant interaction

a) adrien.rohfritsch@sorbonne-universite.fr; between cylinders. More recently, a paper by Linton and Martin $^{7}$ deals with the second-order corrections of effective wavenumbers in elastic media, and derives Twersky's expressions to criticize their relevance. Later, Norris and Conoir ${ }^{8}$ performed the calculation of effective wavenumbers up to fourth order. All these analytical models based on statistical tools are intrinsically limited by the concentration and the geometry of the cluster of cylinders, which is often a half-plane or a slab.

The second strategy emerged at the beginning of the seventies. At that time, new numerical methods were available, offering possibilities to resolve multiple scattering problems. First numerical studies were conducted with two cylinders ${ }^{9,10}$ or circular arrays of cylinders around a point source ${ }^{11}$. Over the years, iterative methods have been developed to calculate the exact solution by using parallel computing. Several methods were designed to resolve problems with particular geometry, such as lattices or uniform distributions in a slab. A wide overview of these numerical methods has been presented recently by Amirkulova and Norris ${ }^{12}$. Among all these methods, the Fast Multipole Method (FMM), proposed by Greengard and Rokhlin ${ }^{13}$ for particle simulations, was later extended for acoustic and electromagnetic scattering calculations. It is worth noting that many efforts have been put on FMM in 3D multiple scattering problems taking into account a large number of spherical particles. More details can be obtained by referring to ${ }^{14,15}$ and the book written by Gumerov and Duraiswami ${ }^{16}$ presenting an upto-date discussion of the FMM including different expansions and convergence analyses. Surprisingly enough, the FFM has received less attention in 2D where it has been implemented by Zhang and $\mathrm{Li}^{17}$ with a special attention 
to phononic crystals.

The objective of this paper is to develop a numerical method of resolution based on the equations of the multiple scattering theory. We focus on demonstrating how the number of non-zero matrix entries can be decreased by introducing a cutoff radius around each cylinder. This radius makes the system sparse and easier to solve using relevant iterative methods. For implementation, we use a library developed to solve large linear sparse systems (Portable, Extensible Toolkit for Scientific Computation, PETSC $\left.^{18-20}\right)$. This library is used with Message Passing Interface (MPI) architecture, which is the key tool to ensure the scalability of the resolution. Indeed, the same implementation works as well on a computer of 24 processors as on a computer of 512 processors: there is no limit to the size of the problem. This implementation allows computation of the most general multiple scattering cases: any number of scatterers, any number of modes (i.e. any frequency) can be treated, as well as any random geometry (with any concentration) or any cylinder type and incident wave type (plane wave, point source, etc.). Validation tests open a comparison between homogeneous (statistical) predictions and our resolution (called MuScat in this paper, for Multiple Scattering). The last part is dedicated to the study of the directivity diagram of a circular cluster of randomly distributed cylinders. In this case computations are perormed with matrices of size $N \times N=85100 \times 85100$ on a computer of 64 processors.

\section{THEORETICAL MODEL}

This section is dedicated to the presentation of the model of multiple scattering of sound waves in two dimensions. The acoustic pressure is written $p e^{-i \omega t}$, and satisfies the Helmholtz equation

$$
\Delta p+k^{2} p=0
$$

where $k=\omega / c$ is the wavenumber and $c$ the speed of sound in the host medium. For the sake of simplicity, the time dependence $e^{-i \omega t}$ is omitted throughout the text. The pressure field is expressed in the coordinate system $(r, \theta)$ which is linked to an arbitrary center $O$. In two dimensions, any wave can be decomposed in the basis of the cylindrical harmonics; that gives for the incident pressure $p_{0}$

$$
p_{0}(\mathbf{r})=\sum_{n=-\infty}^{+\infty} b_{n} J_{n}(k r) e^{i n \theta}
$$

where coefficients $b_{n}$ are the amplitudes of each mode and $J_{n}$ is the Bessel function of order $n$. For instance, for a plane wave propagating along the $x$-axis, the coefficients $b_{n}$ are $(-i)^{n}$. The total sound field in a particular configuration of $N_{s}$ scatterers can be expressed as the sum of the incident wave and the waves scattered by all scatterers

$$
p(\mathbf{r})=p_{0}(\mathbf{r})+\sum_{j=1}^{N_{s}} p_{s}^{(j)}\left(\mathbf{r}_{\mathbf{j}}\right)
$$

Figure 1 indicates the notations used in the following, illustrating that our resolution is not limited to a unique type of cylinder. Eq (3) expresses waves diverging from each cylinder with respect to their own coordinate systems $\mathbf{r}_{\mathbf{j}}=\left(r_{j} \cos \theta_{j}, r_{j} \sin \theta_{j}\right)^{t}$. It follows that

$$
p_{s}^{(j)}\left(\mathbf{r}_{\mathbf{j}}\right)=\sum_{n=-\infty}^{+\infty} A_{n}^{(j)} H_{n}^{(1)}\left(k \mathbf{r}_{\mathbf{j}}\right) e^{i n \theta_{j}}
$$

where $H_{n}^{(1)}$ is the Hankel function of the first kind and order $n$ and the scattering amplitudes $A_{n}^{(j)}$ are the unknowns of the problem. Every scattered wave becomes an incident wave for the other cylinders. Hence, the incident wave on scatterer $j, p_{i n c}^{(j)}$, is written as

$$
p_{i n c}^{(j)}=p_{0}^{(j)}+\sum_{\substack{k=1 \\ k \neq j}}^{N_{s}} \sum_{n=-\infty}^{+\infty} A_{n}^{(k)} H_{n}^{(1)}\left(k r_{k}\right) e^{i n \theta_{k}} .
$$

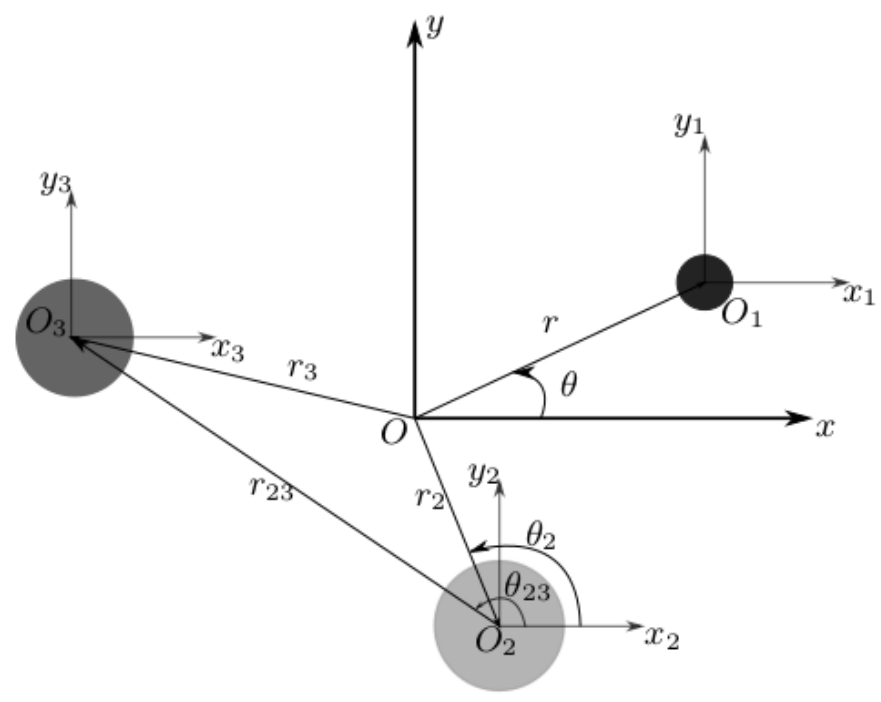

FIG. 1. Arbitrary planar configuration of parallel cylinders.

Using the addition theorem ${ }^{21}$, cylindrical harmonics with origin at $r_{p}$ can be expressed as a sum of cylindrical harmonics with origin at $r_{q}$ thanks to the relations

$$
\left\{\begin{array}{c}
z_{n}\left(k r_{p}\right) e^{i n \theta_{p}}=\sum_{\nu=-\infty}^{+\infty} z_{n-\nu}\left(k r_{p q}\right) e^{i(n-\nu) \theta_{p q}} J_{\nu}\left(k r_{q}\right) e^{i \nu \theta_{q}} \\
\text { if } r_{q}<r_{p q}, \\
z_{n}\left(k r_{p}\right) e^{i n \theta_{p}}=\sum_{\nu=-\infty}^{+\infty} J_{n-\nu}\left(k r_{p q}\right) e^{i(n-\nu) \theta_{p q}} z_{\nu}\left(k r_{q}\right) e^{i \nu \theta_{q}} \\
\text { if } r_{q}>r_{p q},
\end{array}\right.
$$


where $z_{n}$ can either be $J_{n}$ or $H_{n}^{(1)}$, and $\mathbf{r}_{\mathbf{p q}}=\mathbf{r}_{\mathbf{p}}-\mathbf{r}_{\mathbf{q}}$. Substituting Eq (6) in Eq (5) yields

$p_{\text {inc }}^{(j)}=\sum_{\nu=-\infty}^{+\infty} \sum_{n=-\infty}^{+\infty}\left[N_{\nu n}^{(0 j)} b_{n}+\sum_{\substack{k=1 \\ k \neq j}}^{N_{s}} M_{\nu n}^{(k j)} A_{n}^{(k)}\right] J_{\nu}\left(k r_{j}\right) e^{i \nu \theta_{j}}$,

with

$$
\left\{\begin{array}{l}
M_{\nu n}^{(k j)}=H_{\nu-n}^{(1)}\left(k r_{k j}\right) e^{i(\nu-n) \theta_{k j}}, \\
N_{\nu n}^{(0 j)}=J_{\nu-n}\left(k r_{0 j}\right) e^{i(\nu-n) \theta_{0 j}}
\end{array}\right.
$$

Taking into account the scattering coefficients $T_{n}^{(j)}$ of the $\mathbb{T}^{(j)}$ matrix, it follows from Eqs (4) and (7) that we have

$$
A_{n}^{(j)}=T_{n}^{(j)}\left[\sum_{n=-\infty}^{+\infty} N_{\nu n}^{(0 j)} b_{n}+\sum_{\substack{k=1 \\ k \neq j}}^{N_{s}} \sum_{n=-\infty}^{+\infty} M_{\nu n}^{(k j)} A_{n}^{(k)}\right] .
$$

For circular cylinders, the $\mathbb{T}^{(j)}$ matrix is diagonal, with coefficients $\mathbb{T}_{n p}^{(j)}=T_{n}^{(j)} \delta_{n p}$. Its coefficients are calculated imposing continuity of displacements and stress vector at the boudaries of cylinders and fluid. For instance, they are for the case of a soft cylinder of radius $a$

$$
T_{n}^{(j)}=-\frac{J_{n}(k a)}{H_{n}^{(1)}(k a)} .
$$

Note that the cylinders considered in this paper are either soft cylinders (see section IV. A) or elastic cylinders (see all other sections), and that their elastic properties can be chosen by changing $T_{n}^{(j) 22,23}$. Eq (9) can be formulated in a vectorial way as follows

$$
\mathbf{A}^{(j)}=\mathbb{T}^{(j)}\left[\mathbb{N}^{(0 j)} \mathbf{b}+\sum_{\substack{k=0 \\ k \neq j}}^{N_{s}} \mathbb{M}^{(j k)} \mathbf{A}^{(k)}\right] .
$$

If the modal sum is truncated at order $N_{m}, \mathrm{Eq}$ (11) involves $2 N_{m}-1$ equations for each scatterer. The total size of the system is then $N \times N$, with $N=N_{s} \times\left(2 N_{m}-1\right)$ and can be written as

$$
[\mathcal{I}-\mathcal{T} \mathcal{M}] \mathbf{A}=\mathcal{T} \mathbf{E},
$$

with the following notations

$$
\left\{\begin{array}{l}
\mathbf{A}=\left(\mathbf{A}^{(1)}, \mathbf{A}^{(2)}, \ldots, \mathbf{A}^{\left(N_{s}\right)}\right)^{t} \\
\mathbf{E}_{p}=\mathbb{N}^{(0 p)} \mathbf{b} \\
\mathcal{T}_{p q}=\mathbb{T}^{(p)} \delta_{p q} \\
\mathcal{M}_{p q}=\mathbb{M}^{(p q)}\left(1-\delta_{p q}\right) .
\end{array}\right.
$$

Solving the linear system (12) gives all the amplitudes of the waves scattered by the $N_{s}$ arbitrarily distributed scatterers. The calculation of the inverse of the matrix $[\mathcal{I}-\mathcal{T} \mathcal{M}]$ is a very demanding process because of its large size and the fact that it is a dense system. The next part is devoted to our numerical strategy and method to resolve it.

\section{NUMERICAL METHOD}

\section{A. Truncation of the modal sum}

The matrix of the linear system (12) is complexvalued, dense, of size $N \times N$, with $N=N_{s} \times\left(2 N_{m}-1\right)$. The first parameter to set is the number of modes to take into account $\left(N_{m}\right)$, which increases as a function of frequency. A common empirical approach to truncate the modal sum is to choose $N_{m}$ as ${ }^{24}$

$$
N_{m}=\left[k a+\left(1 /(2 \sqrt{2}) \ln \left(2 \sqrt{2} \pi k a \epsilon^{-1}\right)\right)^{2 / 3}+1\right],
$$

where $\mathrm{a}$ is the radius of the scatterers, $[x]$ is the integer part of a real number $x$ and $\epsilon$ is the desired error bound on the scattering amplitudes. This threshold can be insufficient for configurations with very close cylinders. Valier-Brasier and Conoir $^{25}$ recently showed that a large number of modes has to be taken into account in the case of resonant interactions between close scatterers (for instance bubbles or soft cylinders). In this paper, to be sure to converge, we chose to calculate the number $N_{m}$ by resolving a two cylinders problem, with an in-between distance equal to the minimal distance between cylinders in the distribution we want to focus on. Then, starting with $N_{m}=1$ and increasing it more and more, calculation stops when $\left|A_{N_{m}+1}^{1,2}\right|<$ tol and $\left|A_{N_{m}+2}^{1,2}\right|<$ tol simultaneously; tol parameter is set at
$10^{-6}$

\section{B. From dense to sparse systems}

If the size of the matrix becomes large, two problems occur. First of all, there is a possible overflow of the memory space.

A close look at the matrix shows that each line corresponds to the interaction of a scatterer with all other scatterers. But these interactions clearly become smaller as the distance between them increases: the wave scattered by one cylinder does not contribute to the field incoming upon on another scatterer located at a very long distance. That leads to the idea of a cutoff radius $(D)$ around each scatterer, that represents the horizon beyond which cylinders do not interact anymore. This radius is related to the quantities of the problem: the wavelength of the source, the concentration of cylinders, the geometry of the cluster and the elastic properties of the cylinders being the most important.

Imposing this cutoff radius leads to a new problem to solve, written as

$$
[\mathcal{I}-\mathcal{T} \tilde{\mathcal{M}}] \tilde{\mathbf{A}}=\mathcal{T} \mathbf{E}
$$

with $\tilde{\mathcal{M}}$ the new sparse matrix of the problem and $\tilde{\mathbf{A}}$ the new amplitude vector.

Now that a new sparse problem has been constructed, one has to know how to resolve it. 

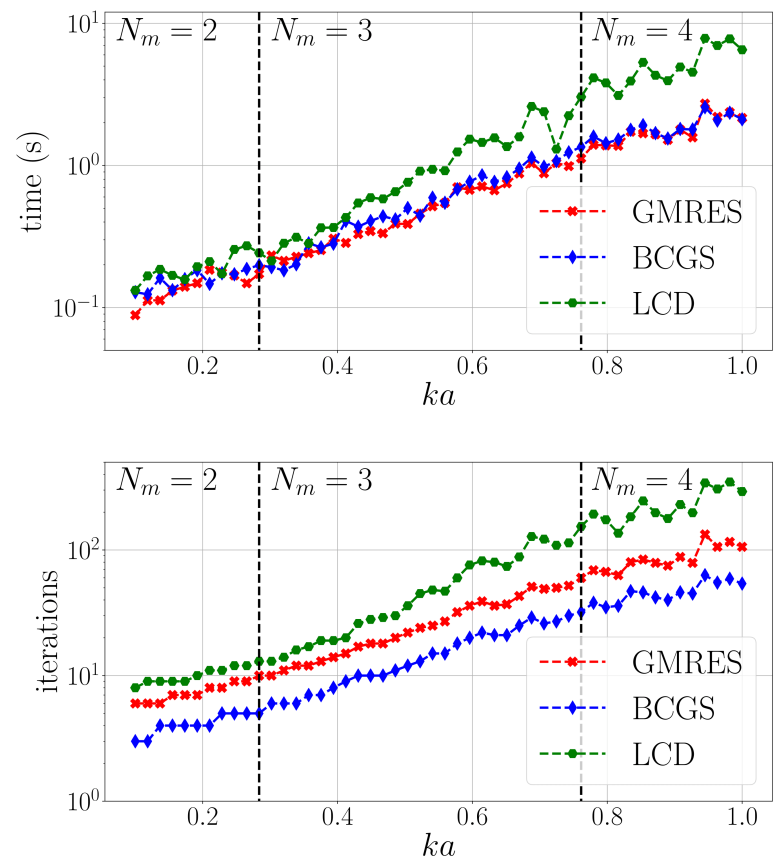

FIG. 2. (color online) Comparison between three iterative methods (GMRES, BCGS and LCD), in terms of convergence time (above) and number of iterations (below). Calculations are performed on 480 steel cylinders of radius $a=1 \mathrm{~mm}$ randomly distributed in water, with a global concentration of $\phi=8 \%$.

\section{Iterative methods designed to work through Message Passing Interface}

To resolve such huge sparse linear systems, direct methods are known to be inefficient, because of their time and memory costs: for instance, Gauss-Jordan method requires $\mathcal{O}\left(N^{3}\right)$ operations, and LU decomposition $2 N^{3} / 3^{12}$. Iterative methods are then more suitable. Those based on Krylov subspaces have concentrated a lot of efforts through the last two decades ${ }^{24,26,27}$. A set of these methods is computed in PETSC's library ${ }^{18-20}$, which we chose here to perform the resolution.

This library works using Message Passing Interface (MPI). This parallel computing architecture is characterized by the fact that each processor has its own memory storage. Data communication is performed from one processor to another. An important advantage of this architecture is the scalability of the resolution. Indeed, after implementing the resolution on a computer of 64 processors, one can immediatly launch it on computers of 24 or 512 processors without any difficulty, and therefore increase the maximum size of problems that can be resolved.
For each matrix type, a particular iterative method has to be chosen, because it has to take into account the global and local properties of the matrix $[I-\mathcal{T} \tilde{\mathcal{M}}]$. In our case, it is a complex-valued asymmetrical matrix. Many studies are limited to cases with periodic lattices, for which the problem is easier to solve. For instance, Biwa et $a .^{28}$ focused on the propagation of shear waves in periodic lattices (even though they presented the general model), studying propagation in composite media. More recently, Amirkulova and Norris ${ }^{12}$ compared the LAPACK and TOEPLITZ libraries, and treated problems of phononic crystals composed of a few hundred cylinders.

The objective of the study here is to resolve the problem for the most general case, for which the matrix does not have particular symmetries. Three of the suitable methods for such matrices were studied in terms of convergence rate: Stabilized version of BiConjugate Gradient (BCGS), Generalized Minimal Residual method (GMRES) and Left Conjugate Direction (LCD). The preconditionner Block Jacobi is chosen for the three methods. Two comparisons are presented in Figure 2 for a random distribution of 480 steel cylinders with a concentration of $\phi=8 \%$ : time needed to reach converge (above) and number of iterations to converge (below). Here, the sparsity of the system is such that approximately $20 \%$ of the interactions between scatterers are taken into account: all cylinders interact with the closest $20 \%$ cylinders.

GMRES is chosen to perform all the simulations throughout this paper, because of its better resolution speed in a classical frequency range. One can note that the BCGS method gives close results in terms of time, and better results in terms of number of iterations (but each iteration takes more time).

Numerical methods for solving large linear sparse systems are now well established.

\section{Controlling the error introduced by the cutoff radius}

Now that one knows how to make the system sparse and that an efficient method to solve sparse asymmetrical systems has been chosen, the error created by the introduction of the cutoff radius has to be quantified: the question is now how to choose $D$ and what error is created by this choice.

This error is evaluated through the error made on the acoustic field (which is directly related to the amplitudes themselves, but closest to physical considerations). Several simulations are performed on circular random distributions of steel and epoxy cylinders in water. This geometry is chosen because it minimises the number of scatterers close to the domain boudaries. To cancel boundary effects, no cutoff radius is applied to cylinders too close from the boundary (i.e. for which the exclusion circle is totally included in the cluster).

The radius of each distribution is six times larger than the wavelength of the incident field, which is a plane wave. 
The error created by the cutoff radius is calculated by the relation

$$
\Delta_{E}=\sqrt{\left(\frac{\sum_{r}^{N_{r}} \sum_{\theta}^{N_{\theta}}|p(r, \theta)-\tilde{p}(r, \theta)|^{2}}{\sum_{r}^{N_{r}} \sum_{\theta}^{N_{\theta}}|p(r, \theta)|^{2}}\right)},
$$

where $p(r, \theta)$ is the exact pressure field (calculated by resolving the dense system) and $\tilde{p}(r, \theta)$ is the approximate pressure field. $N_{r}$ is the number of sampling points in radial direction, and $N_{\theta}$ in angular direction. These two parameters, as soon as they are taken large enough (few tens by wavelengths in our case) do not impact the value of $\Delta_{E}$. Here, we chose to impose $\Delta_{E}=10 \%$. The calculations are performed for different frequencies and concentrations in such a way that the dense version of the matrix can fit in the memory of a $125 \mathrm{~Gb}$ computer. The maximum values of the cutoff radius, normalized by the wavelength, to obtain an error equal to $10 \%$ are given in Figures 3, for steel cylinders and epoxy cylinders, as a function of $\phi \in[5 \%, 20 \%]$ and the frequency parameter $k a \in[0.1,1.0]$. Twenty-four points have been calculated in frequency and twelve in concentration.

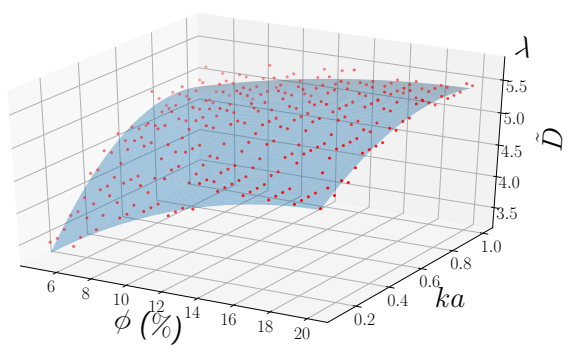

(A)

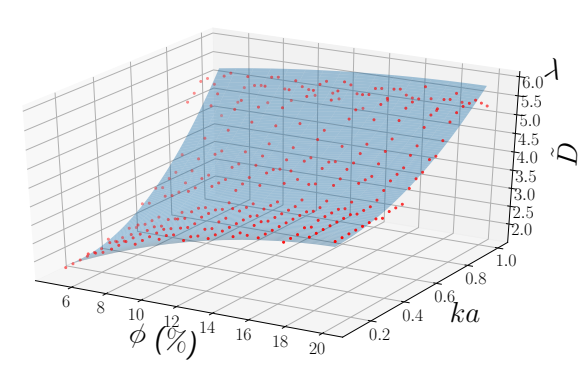

(B)

FIG. 3. (color online) Maximum values for cutoff radius for which the error is $\Delta_{E}=10 \%$, as a function of frequency and concentration, for two types of elastic cylinders: (A) steel $\left(\rho_{c}=7850 \mathrm{~kg} \cdot \mathrm{m}^{-3}, c_{L}=5700 \mathrm{~m} \cdot \mathrm{s}^{-1}, c_{T}=3000 \mathrm{~m} \cdot \mathrm{s}^{-1}\right)$ and (B) epoxy $\left(\rho_{c}=1200 \mathrm{~kg} \cdot \mathrm{m}^{-3}, c_{L}=1500 \mathrm{~m} \cdot \mathrm{s}^{-1}, c_{T}=800\right.$ $\left.\mathrm{m} . \mathrm{s}^{-1}\right)$.

This surface can be fitted by a function of two parameters. For each study, one can then determine which cutoff radius should be imposed in order to have a tolerable error. Writing $\tilde{D}(k a, \phi)=D(k a, \phi) / \lambda$ as the products of two polynomials of degree deg

$$
\begin{aligned}
\tilde{D}(k a, \phi) & =\left[\sum_{i=0}^{\operatorname{deg}} a_{i}(k a)^{i}\right]\left[\sum_{j=0}^{\operatorname{deg}} b_{j} \phi^{j}\right] \\
& =\sum_{i=0}^{\operatorname{deg}} \sum_{j=0}^{\operatorname{deg}} c_{l}(k a)^{i} \phi^{j}, \quad \text { with } l=j+i \operatorname{deg}
\end{aligned}
$$

one can use a least square method to determine appropriate values for coefficients. In the case of steel cylinders, Figure $3(\mathrm{~A})$ shows that $\tilde{D}$ behavior is quite smooth. In this case, interpolated surface gives good approximation with $\operatorname{deg}=2$. Coefficients are given in Table I. In the case of epoxy cylinders (B), an elastic resonance around $k a=0.88$ makes the behavior much more complicated, for which deg $=4$ has to be chosen in order to get a correct interpolation. For the sake of legibility, the 23 coefficients for this second surface are not given in the text.

\begin{tabular}{ccccccccc}
\hline \hline$c_{0}$ & $c_{1}$ & $c_{2}$ & $c_{3}$ & $c_{4}$ & $c_{5}$ & $c_{6}$ & $c_{7}$ & $c_{8}$ \\
1.6 & 29.0 & 7.1 & -48.8 & -72.6 & -4.3 & 29.6 & 130.5 & -81.1 \\
\hline \hline
\end{tabular}

TABLE I. Coefficients of surface of cutoff radius calculated for steel cylinders.

We chose here to link the dimension of cutoff radius to the wavelength. Other lengths can be chosen, such as radius $a$, elastic mean free path $l_{e}$, cluster size $R_{c}$ or even mean distance between cylinders $d$ (depending on the concentration). The elastic mean free path can appear as a very relevant quantity because of its strong dependency on frequency and concentration, but there are two main problems with it. The first problem is related to cylinder resonances. The second problem is linked with the fact that, outside of low concentration regimes, it is difficult to calculate the elastic mean free path with good precision (note that for low concentration, Eq (20) in last section gives the standard expression). Here, the wavelength is chosen to scale the cutoff radius. Our choice is motivated by the fact that the wavelength is the main quantity playing a role in the behavior of the Hankel functions, which drives the scattering. Zhang and $\mathrm{Li}^{17}$ also used this quantity to scale their groups of scatterers. Another proof of the complex dependency of cutoff radius on frequency follows in the next part of this article.

The choice of the threshold $\Delta_{E}=10 \%$ is arbitrary, other values are of course possible. To get a physical meaning for this value, a circular, randomly distributed distribution of 2130 steel cylinders $\left(\rho_{c}=7850 \mathrm{~kg} \cdot \mathrm{m}^{-3}\right.$, $\left.c_{L}=5700 \mathrm{~m} . \mathrm{s}^{-1}, c_{T}=3000 \mathrm{~m} \cdot \mathrm{s}^{-1}\right)$ in water $(c=1500$ 
m.s ${ }^{-1}, \rho=1000 \mathrm{~kg} . \mathrm{m}^{-3}$ ) is considered. The global concentration is $\phi=15 \%$. A plane wave propagates towards the $x$-direction (from left to right), with frequency $f=46.8 \mathrm{kHz},(k a=0.2)$.

Figures 4 (A), (B), (D), (F) and (H) show the scattered energy field (in $\mathrm{dB}$ ), in four cases: (A) shows the exact field (obtained by resolution of the dense problem), and (B), (D), (F) and (H) three sparse systems, with smaller and smaller cutoff radius $((\mathrm{F})$ corresponding to the Born approximation case, for which no interaction is taken into account). (B) corresponds to the rate proposed in this work $\Delta_{E}=10 \%$. All the quantities are reported in Table II.

\begin{tabular}{cccc}
\hline \hline$D(\mathrm{~m})$ & $\Delta_{E}(\%)$ & $\%$ of interactions & Figures: field, error \\
\hline 0.19 & 10.0 & 96.0 & $(\mathrm{~B}),(\mathrm{C})$ \\
0.15 & 22.0 & 77.0 & $(\mathrm{D}),(\mathrm{E})$ \\
0.09 & 49.0 & 39.0 & $(\mathrm{~F}),(\mathrm{G})$ \\
0.001 & 91.0 & 0.0 & $(\mathrm{H}),(\mathrm{I})$ \\
\hline \hline
\end{tabular}

TABLE II. Energy error, for four different cutoff radius $D$ on the same distribution.

The considered distribution has a radius of $R_{c}=0.1$ $\mathrm{m}$, which corresponds to $100 a$. This gives a ratio $k R_{c}=20$. Considering the cluster cylindrical form, we observe here a scattering behavior similar to the one of a single cylinder scattering at high frequency regime for which most of the energy goes forward. The error on the acoustic fields is represented in Figures 4 (C), (E), (G) and (I). Although $\Delta_{E}$ takes large values, one can observe how weak the error on the field is. This is due to the fact that the error estimator $\Delta_{E}$ includes the error made on the total areas (which are two times bigger than the considered distribution). For both cases (C) and $(\mathrm{E})$, the error is almost everywhere below $15 \mathrm{~dB}$, even if $\Delta_{E}=22 \%$ for case (E). It should be pointed out that the choice of $\Delta_{E}$ can depend on other parameters, for instance the geometry of the cluster. In our case corresponding to a cylindrical cluster, most of the energy is located on the forward scattering region.

\section{VALIDATION TESTS}

This section is devoted to validation cases for which MuScat simulations are compared to other simulations performed using other calculation methods. First, a numerical calculation based on Discontinuous Galerkin Method is performed, on situations with a few soft cylinders. Then, a comparison with Foldy's Model ${ }^{29}$ gives very good results, in terms of effective parameters, for low concentration $(\phi=6 \%)$ and frequency range $k a \in[0,1]$.

\section{A. Comparison with numerical simulations of the wave equa- tion}

Time-domain calculations based on the wave equation is another strategy to investigate multiple scattering problems. For instance, Pennec et al. ${ }^{30}$ performed Finite Difference Time Domain simulations to study multiplexing and demultiplexing of waves in waveguides. Chekroun et al. ${ }^{31}$ used a time simulation to compute the effective wave numbers of heterogeneous media in order to compare them with the effective medium theories we listed in the introduction of this paper. These methods are limited by the number of scatterers, due to mesh size limitation. The idea of this section is to compare the scattered field of simple configurations of soft scatterers, calculated by MuScat and by the Discontinuous Galerkin calculator Paradigm ${ }^{32,33}$.

Paradigm is based on time simulation. The source defined in both simulation is a plane wave and the radius of the cylinders is $a=1 \mathrm{~mm}$. Ideal plane waves are never reached in time domain simulations, because that means infinite extension. To stay free from diffraction consideration, we decided to compare only the scattering field rather than the total field. Two time simulations have been performed for each case presented here: one with cylinders and the other without (the second one is a free space simulation). In this way, substracting both fields gives the scattering part of the total field. Afterwards, a frequency treatment is performed to get the harmonic picture of the field, instead of the time simulation.

Two different configurations are presented here. One is composed of four cylinders placed on the corners of a square, $1 \mathrm{~cm}$ by $1 \mathrm{~cm}$. The other is composed of 6 randomly distributed cylinders. This last case is interesting because cylinders very close to each other are considered. Figures 5 (A),(B) show the scattered fields on two different space lines for two configurations. The space line is represented in green at the top left corner and it is parallel (a) or perpendicular (b) to the propagation direction of the source. The frequency of the source is $k a=0.7$ for $(\mathrm{A})$ and $k a=1.06$ for (B). MuScat and Paradigm agree with a very good precision in both cases. These are the first tests proving the validity of the implemented method.

More than just a validation case, it is also an interesting case of comparison between two different models, one using discretization of elastodynamics equations (Paradigm) and the other being only based on acoustical quantities, where the mechanical aspects are all included in the $\mathbb{T}$-matrix of each cylinder.

\section{B. Comparison with the Independant Scattering Approxima- tion (ISA)}

For low concentrations of scatterers, effective medium theories are often used to calculate the effective celerity and the effective attenuation of the coherent wave through the heterogeneous medium. The idea of the current section is to compare the effective parame- 


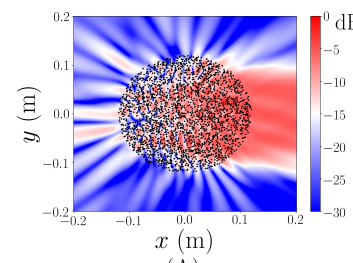

(A)

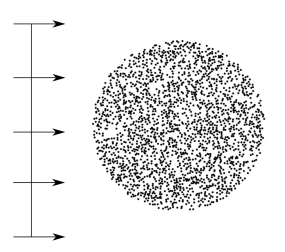

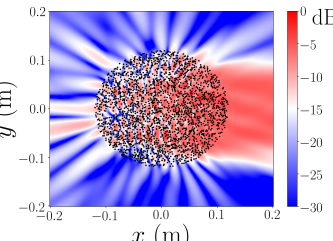

(B)

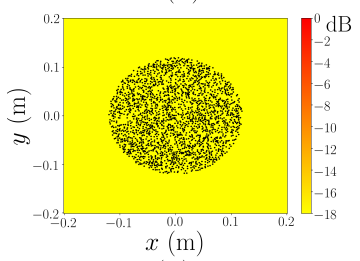

(C)

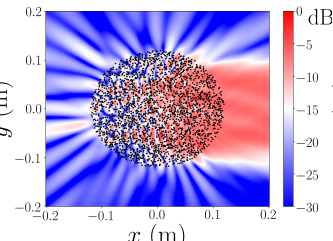

$x(\mathrm{~m})$
(D)

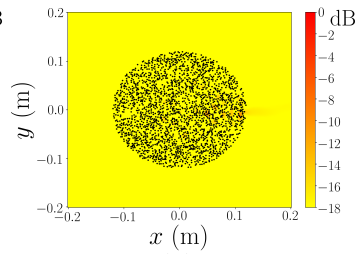

(E)

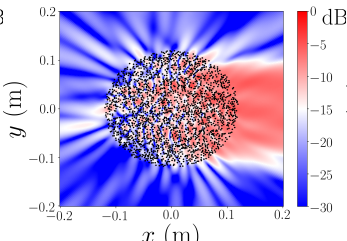

$x(\mathrm{~m})$
$(\mathrm{F})$

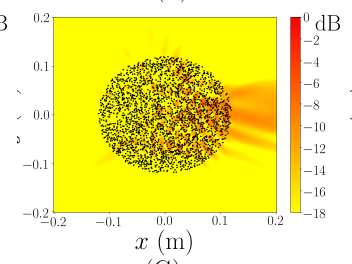

(G)

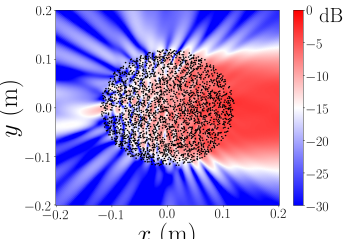

$x(\mathrm{~m})$
$(\mathrm{H})$

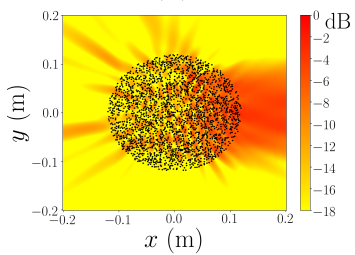

(I)

FIG. 4. (color online) Acoustical energy fields calculated on circular randomly distributed distributions $(\phi=15 \%, k a=0.2)$. A plane wave is propagating from left to right. Figures on the top represent the acoustic energy normalized by the maximum of (A). (A) is calculted thanks to the dense system (12), and the others with (14), with smaller and smaller cutoff radius, reported on Table II. Figures at the bottom represent the energy error introduced by the cutoff radius, with respect to (A).

ters given by Foldy's model and those given by MuScat. Let us consider a rectangular geometry of size $H \times h, H$ being the dimension towards the $y$-axis and $h$ towards the $x$-axis, as presented in Figure 6 . A plane wave is chosen as source and propagates towards the $x$-axis. At position $x=h$, one can write the pressure as

$$
\begin{cases}p_{0}=A e^{j k h} & \text { in homogeneous medium, } \\ p_{1}=A e^{j k_{\text {eff }} h} & \text { in heterogeneous medium. }\end{cases}
$$

The effective wave number is complex and decomposed as $k_{\text {eff }}=\omega / c_{\text {eff }}+j \alpha_{\text {eff }}$, where $\alpha_{\text {eff }}$ is the effective attenuation and $c_{\text {eff }}$ the effective phase velocity. Considering that the phase delay $\arg \left(p_{1} / p_{0}\right)$ is simply given by $\omega h\left(1 / c_{\phi}-1 / c_{0}\right), \alpha_{\text {eff }}$ and $c_{\text {eff }}$ are calculated as follows

$$
\left\{\begin{array}{l}
\alpha_{\mathrm{eff}}=-\frac{1}{h} \ln \left(\left|\frac{p_{1}}{p_{0}}\right|\right), \\
c_{\mathrm{eff}}=\frac{\omega h}{\frac{\omega h}{c_{0}}+\arg \left(\frac{p_{1}}{p_{0}}\right)} .
\end{array}\right.
$$

Foldy's model gives an expression for the effective wavenumber ${ }^{8}$.

$$
k_{\text {eff }}^{2}=k^{2}-4 i n_{0} \sum_{n} T_{n},
$$

where $n_{0}$ is the number of cylinders by square meter and $T_{n}$ the scattering coefficients of one single cylinder. Expressions (18) (used for calculation with MuScat) and (19) are compared numerically.

Calculations with MuScat are performed for randomly distributed distributions of steel cylinders $\left(\rho_{c}=7850 \mathrm{~kg} \cdot \mathrm{m}^{-3}, c_{L}=5700 \mathrm{~m} . \mathrm{s}^{-1}, c_{T}=3000 \mathrm{~m} . \mathrm{s}^{-1}\right)$ in water $\left(c=1500 \mathrm{~m} \cdot \mathrm{s}^{-1}, \rho=1000 \mathrm{~kg} \cdot \mathrm{m}^{-3}\right)$. The concentration is $\phi=6 \%$, which is low enough to consider Foldy's model as a reference. The radius of the cylinders is $a=400 \mu \mathrm{m}$. The slab sizes are $H=60 \mathrm{~cm}$ and $h=18 \mathrm{~mm}$ (each slab is composed of 1289 cylinders). A plane wave propagates towards $x$-direction. To be as close as possible to experimental conditions, the acoustic field is calculated on the surface $H_{c}=0.2 \mathrm{H}$ on which the average is performed. In order to stay free from diffraction considerations (which can occur near the boundaries), the average on 30 different slabs is then calculated instead of moving the measurement line towards the vertical direction. Calculations are performed with $N_{m}=3$, which is relevant for the frequency range investigated $0 \leq k a \leq 1$.

Figures 7 (A) and (B) show the dependency of the effective attenuation $\alpha_{\text {eff }}$ and phase velocity $c_{\text {eff }}$ on frequency. Even if the cylinders (steel cylinders) are more rigid than the host medium (which is water), the effective phase velocity is predicted to be lower than in the host medium for the entire frequency range. Considering the phase velocity as being controlled by a competition between the stiffness of the medium and its density, it is interesting to note here that the effective density increases more than the effective stiffness, which explains why the coherent wave front slows down.

For both parameters, results calculated using the Born approximation are also reported. The Born approximation only gives good results for very low frequencies $(k a<0.2)$.

Otherwise, the agreement is excellent when using the cutoff radius ( $D=D(\lambda)$, dark green line) previously calculated. This shows that the surface calculated and presented above can be successfully used in other 


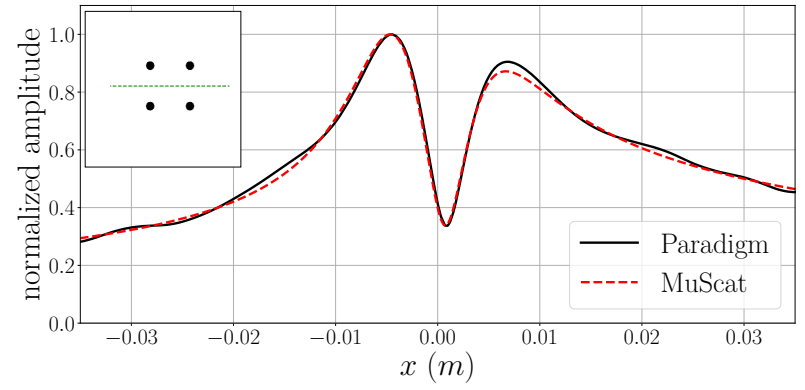

(A)

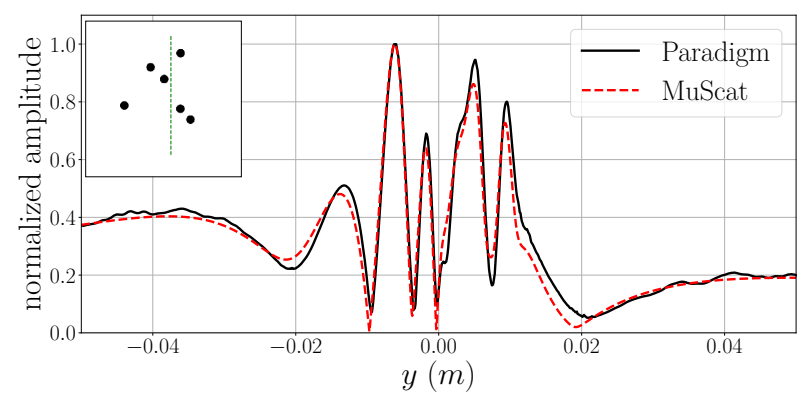

(B)

FIG. 5. (color online) Comparison between the absolute value of the scattered pressure field calculated by Discontinuous Galerkin Method (black lines) and by MuScat (red lines) on a space line represented on green on the top left corner with respect to the positions of the cylinders.

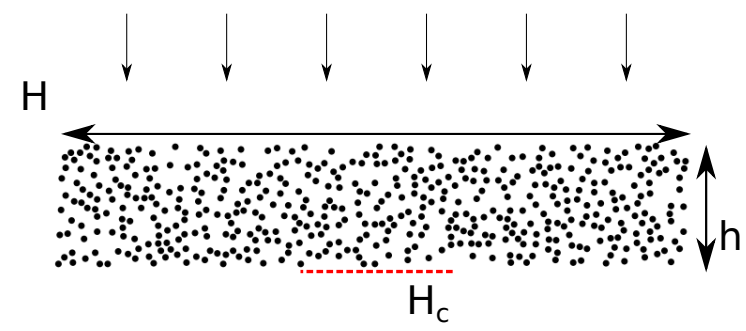

FIG. 6. (color online) Configuration used to extract effective parameters of random distributions with MuScat.

configurations, which differ by the geometry and by the quantities calculated. Considering the $D=2 \lambda$ curve, it is worth noting that the phase velocity does not fit with Foldy's predictions for $k a>0.5$. This shows that the wavelength dependency can be quite complicated and very different from a linear behavior.

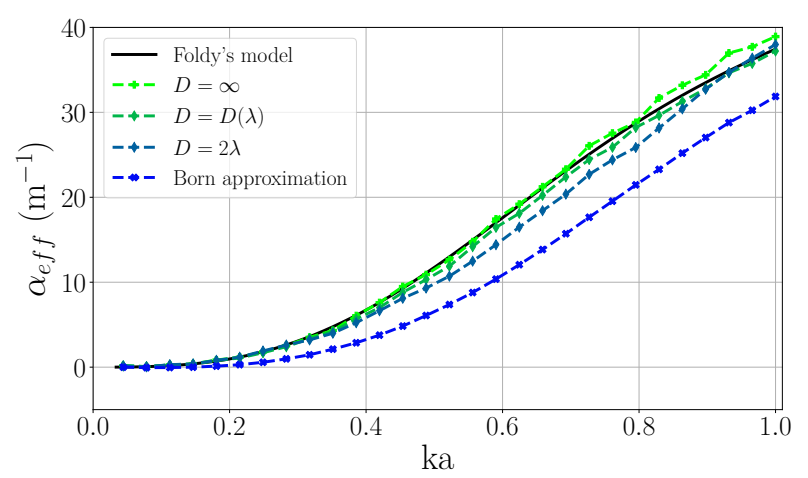

(A)

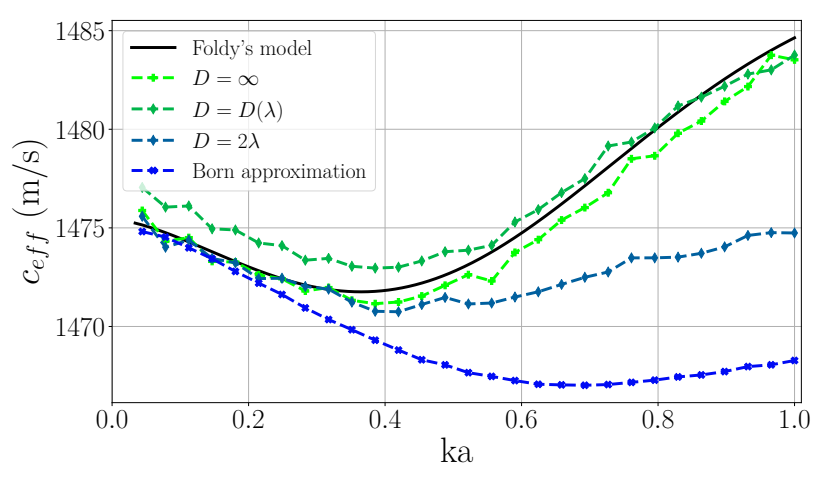

(B)

FIG. 7. (color online) Effective attenuation (A) and phase velocity (B) calculated with MuScat with different cutoff radii.

The tests presented in this section clearly show that the implementation is validated for the tested frequencies and concentrations.

\section{RANDOMLY DISTRIBUTED MEDIA - COMPARISON BETWEEN AVERAGE PROCESS CALCULATED WITH MUSCAT AND BY HOMOGENIZATION}

The main interest of homogenization theories is to compute the effective parameters associated with multiple scattering media, not only the effective wave number, but also density and stiffness ${ }^{34}$. Basically, there are two interests in calculating effective parameters. Either we look for exotic behaviors such as for example negative refraction and cloaking, leading to the investigation of acoustic metamaterials, such as those that have appeared on the scene in the last few years ${ }^{35}$. In such cases, we focus on unusual concepts such as negative density and negative compressibility. Or we use the effective parameters to build a homogenized/effective medium which has the same physical behavior as the multiple scattering medium. This is what was done previously in section IV when the multiple scattering slab was assimilated to an 
effective slab in order to calculate $k_{\text {eff }}$.

The question in this paper is: does the assimilation between multiple scattering and effective media carried out with a slab still work for a cluster having another geometry?

It should be noted that $k_{\text {eff }}$ is calculated by comparing the two transmission coefficients associated with the multiple scattering and effective media. What about the reflection? Reflection coefficients do not enable this objective to be achieved because the coherence manifests itself through the coherent backscattering, which is another physical phenomenon. Coherent waves result from the average of all the acoustic waves traveling from one cylinder to another in the same direction (that of the incident plane wave), and the coherent backscattering is due to reflected paths that are traveled twice in one or other directions ${ }^{36}$. It should also be noted that, even in transmission, it is not possible to assimilate the multiple scattering transmission coefficient $T_{\mathrm{MS}}$ to the effective transmission coefficient $T_{\text {eff }}$ if the slab thickness $h$ is greater than the elastic mean free path $l_{e}$. This quantity is usually defined by the relation ${ }^{37}$

$$
l_{e}=\frac{1}{2 \alpha_{\mathrm{eff}}} .
$$

If $h>l_{e}$, the coherent wave is too attenuated to propagate through the slab and the transmitted field becomes diffusive in nature. Furthermore, if it is well established that $T_{\text {eff }} \approx T_{\mathrm{MS}}$ for $h>l_{e}$ at normal incidence, this result has never been established at oblique incidence. At oblique incidence, a question remains open: is there a refraction effect obeying the Snell-Descartes laws at the interface between a homogeneous and a multiple scattering medium? At normal incidence, the wave front of the incident wave coincides with the interface; this is a favorable situation to generate a coherent wave. At oblique incidence, all the waves do not have the same phase reference when they are excited. There is a phase delay that depends on the angle of incidence. In such a case, how is the coherence built? What is the direction of propagation of the coherent wave?

It is these first elements of analysis that led us to consider a multiple scattering cluster more complex than the slab. We have chosen a cluster of cylindrical shape because it has a geometry simple enough to be homogenized and because its radius of curvature is not infinite. It follows that the angle of incidence between the direction of propagation of the incident plane wave and the surface of the cluster range from 0 to $\pi / 2$. Moreover, it is expected that the backward and forward scattering reveal different and interesting behaviors. The purpose of this last part is to use the effective parameters in order to compare the wave propagation through the cylindrical multiple scattering cluster and the homogenized one. It is worth noting that similar studies have been investigated by Torrent and Sánchez-Dehesa ${ }^{38}$ and Reyes-Ayona et al. ${ }^{39}$ in the case of cylindrical clusters made up of two dimensional sonic crystals and in the case of random clusters of cylinders ${ }^{40}$ with the idea of identifying effective parameters at low frequencies.

The diameter $D_{c}=2 R_{c}$ of the cluster is given in function of the cylinder radius $a=1 \mathrm{~mm}$ and the elastic mean free path $l_{e}$. Two different cases are considered which correspond to the parameters given in Table III. The concentration is $\phi=10 \%$ and the source frequency is fixed at $k a=0.3$. The concentration is a bit higher than the one considered in the previous section but still low enough to consider Foldy's predictions as valid. The physical parameters of the homogenized cylinder are given by $c_{L}=c_{\mathrm{eff}}, \alpha=\alpha_{\mathrm{eff}}$ and $\rho_{\mathrm{eff}}=\phi \rho_{c}+(1-\phi) \rho$. For

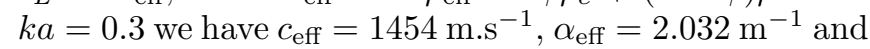
$\rho_{\text {eff }}=1690 \mathrm{~kg} \cdot \mathrm{m}^{-3}$. The effective wavenumber is given by $k_{L}=\omega / c_{\text {eff }}+j \alpha_{\text {eff }}$ and the scattering coefficients $T_{n}$ of the homogenized cluster are the following

$$
T_{n}=\frac{\frac{J_{n}\left(k R_{c}\right)}{J_{n}\left(k_{\mathrm{eff}} R_{c}\right)}-\frac{\rho_{\mathrm{eff}} k J_{n}^{\prime}\left(k R_{c}\right)}{\rho k_{\mathrm{eff}} J_{n}^{\prime}\left(k_{\mathrm{eff}} R_{c}\right)}}{\frac{\rho_{\mathrm{eff}} k H_{n}^{\prime(1)}\left(k R_{c}\right)}{\rho k_{\mathrm{eff}} J_{n}^{\prime}\left(k_{\mathrm{eff}} R_{c}\right)}-\frac{H_{n}^{(1)}\left(k R_{c}\right)}{J_{n}\left(k_{\mathrm{eff}} R_{c}\right)}}
$$

It is important to note here that the scattering by homogenized clusters falls within the framework of the high frequencies because $k R_{c}=36.9$ in the first case $\left(R_{c}=\right.$ $\left.l_{e} / 2\right)$ and $k R_{c}=123.6$ in the second one $\left(R_{c}=1.675 l_{e}\right)$. The convergence of the modal series requires therefore to take into account up to $N_{m}=45$ and $N_{m}=135$ modes of vibration respectively. This shows that the vibration behavior of clusters can be very complicated due to all the interactions that occur at the microscopic level. For the multiple scattering simulations, the average is performed over twenty different random distributions. We calculate the total scattered energy; the directivity diagrams are plotted at a radial distance $l=1.25 R_{c}$. Normalizations are performed from the maximum of energy given by MuScat simulations, and the dynamic range is fixed at $-15 \mathrm{~dB}$ (except for the Figure 11(B) for which it is $-30 \mathrm{~dB}$ ).

\begin{tabular}{cccccc}
\hline \hline & $D_{c} / a$ & $D_{c} / l_{e}$ & $N_{s}$ & $N_{m}$ & $N \times N$ \\
\hline i) & 246 & 1.0 & 1516 & 3 & $7580 \times 7580$ \\
ii) & 824 & 3.35 & 17020 & 3 & $85100 \times 85100$ \\
\hline \hline
\end{tabular}

TABLE III. Cluster dimensions considered for the multiple scattering simulations for a concentration $\phi=10 \%$ and a frequency of $k a=0.3$.

Figure 8 shows results for an incident plane wave propagating from left to right. In Figures (C), (D) the construction of coherent waves resulting from the averaging process appears very clearly. The averaging process is fully effective only from a certain distance away from the boundary of the cylindrical cluster, which is enlightened by the incident plane wave. The place where the coherent wave is generated describes a semi-circle and makes a boundary layer appear on the left side of the cluster, where the multiple scattering is not coherent. 
This effect, which has already been discussed by Linton and Martin ${ }^{21}$, could be quantified from simulations by MuScat.

In Figures (A), (C), for which the diameter is equal to the elastic mean free path, the coherent wave seems to propagate through the cluster as a bulk wave. These figures show a broadly satisfactory agreement between homogenized and numerical simulations. However, if Figure (E) shows a very good agreement in forward scattering, the situation is very different for the backward scattering. This difference can be explained in a fairly simple way. The homogenized simulation assumes that the coherent wave occupies the whole cluster, which is not the case since we have brought to light the existence of a boundary layer (close to the reflection) where the multiple scattering is not coherent. It should also be noted that the forward scattering is very strong, more than $15 \mathrm{~dB}$ higher than the backscattering.

In Figures (B), (D), the elastic mean free path $l_{e}$ is much smaller than the cluster diameter. As shown in Figure (D) the coherent wave is attenuated before reaching the opposite side of the cluster. In this case, we expect to see a difference between homogenized and numerical simulations, even in forward scattering. Surprisingly, this is not what is observed in Figure $(\mathrm{F})$, where the agreement is excellent in forward scattering. In this case, the coherent wave does not propagate through the cluster, as previously studied, but around the cluster. It seems that coherent waves follow the cluster curvature in a clockwise and anticlockwise direction. Can we talk about surface coherent waves? We do not really have a way of analyzing this surface phenomenon, but we can choose another source in order to verify whether or not it still exists.

Figure 9 shows results for a point source located on the axis $y=0$ at a distance of $1.1 R_{c}$ on the left of the cluster. The elastic mean free path $l_{e}$ is much smaller than the cluster diameter. The previous result is fully confirmed, Figures (A), (B) show that most of the energy is radiated from the boundary of the cluster. Homogenized and multiple scattering models seem to give the same results, but, even if the directivity patterns have the same shape, a difference of approximately $10 \mathrm{~dB}$ is visible on all directions in directivity diagrams ( $c f$. Figure $(\mathrm{C}))$. This difference can be explained by the fact that the wave front of the coherent wave is cylindrical, as we can see in Figure (B). So the wave number of the homogenized cluster, which is calculated for a coherent wave with a plane wave front, is probably not well adapted to the situation.

In conclusion, all the results show that homogenization theories are not fully adapted in order to describe the multiple scattering by a cluster of cylindrical shape. In particular, the backward scattering is far from being well described. However, the forward scattering seems always to yield good results if the incident wave is a plane wave, whatever the value of the elastic mean free path compared to the cylindrical cluster diameter.

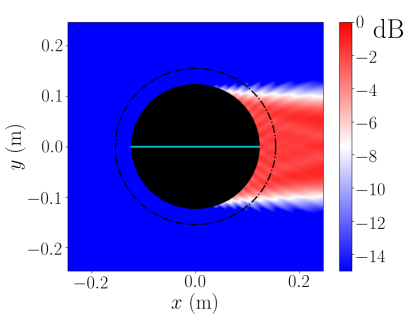

(A)

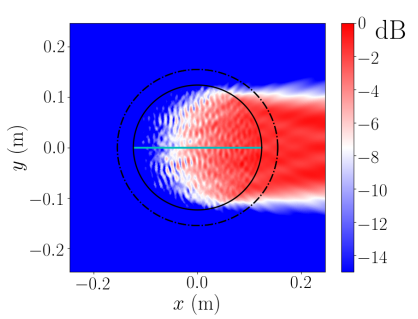

(C)

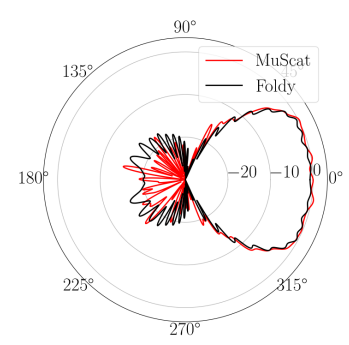

(E)

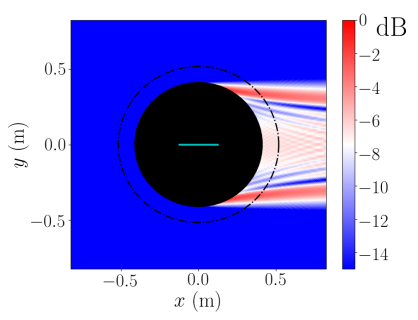

(B)

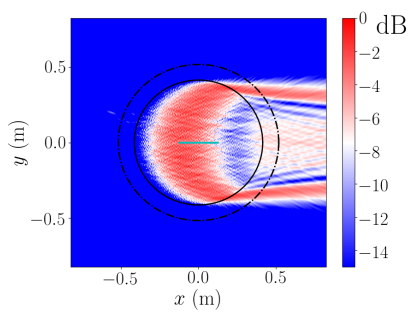

(D)

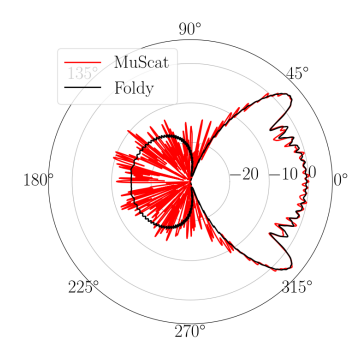

(F)
FIG. 8. (color online) Scattered energy, calculated by homogenization of the heterogeneous medium $((\mathrm{A}),(\mathrm{B}))$ and by MuScat $((\mathrm{C}),(\mathrm{D}))$. The cylindrical distribution has a radius $R_{c}=123 a$ (left) or $R_{c}=412 a$ (right), which correspond to $R_{c}=l_{e} / 2$ (left) or $R_{c}=1.625 l_{e}$ (right). Directivity diagrams (E), (F) are calculated on doted lines indicates in figures above. Source is a plane wave propagating from left to right. Cyan lines in the middle indicated the size of $l_{e}$.

\section{CONCLUSION}

We have implemented an innovative resolution method to treat the multiple scattering of sound in two dimensions. Our resolution method can deal with any situation, with many randomly distributed cylinders, combining numerous different $\mathbb{T}$-matrices and a very large number of cylinders. We have shown applications with matrices of sizes bigger than $80000 \times 80000$ for random positions. The scalability of the resolution method allows us to think about considering much bigger problems. The next step of this work will deal with generalising this method in three dimensions and efforts will be concentrated on comparison with experimental data. 


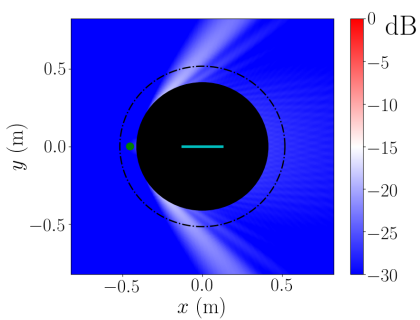

(A)
(B)
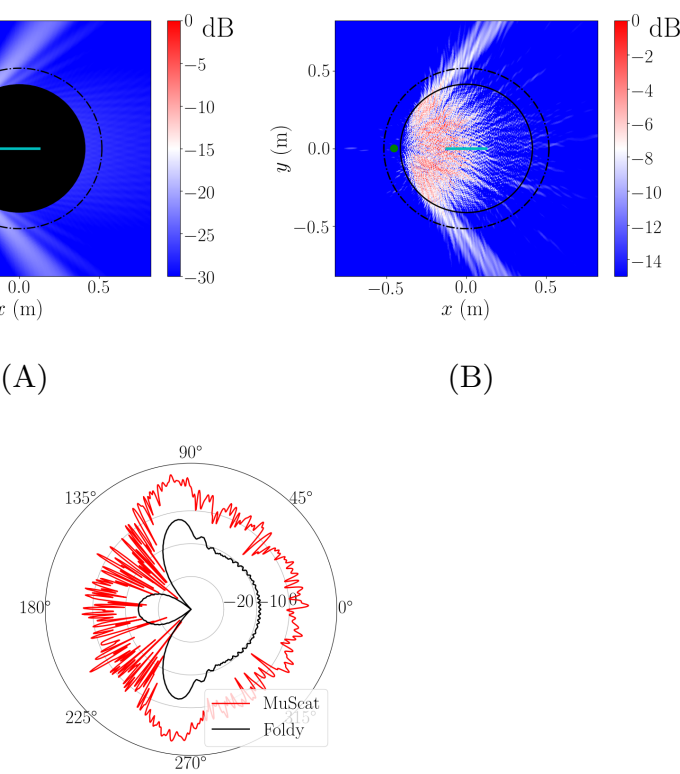

(C)

FIG. 9. (color online) Scattered energy, calculated by homogenization of the heterogeneous medium (A) and by MuScat (B). The cylindrical distribution has a radius $R_{c}=412 a$, which correspond to $R_{c}=6.7 l_{e}$. Directivity diagram $(\mathrm{C})$ is calculated on doted lines indicates on figures above. Source is a point source located on position $\left(1.1 R_{c}, 0\right)$ (green point). Cyan lines in the middle indicate the size of $l_{e}$

\section{ACKNOWLEDGMENTS}

The authors wish to thank $\mathrm{PhD}$ student Pierre Massé (STMS-IRCAM) for his careful reading of this manuscript.

${ }^{1}$ F. Zàviška, "über die Beugung elektromagnetischer Wellen an parallelen, unendlich langen Kreiszylindern", Annalen der Physik 345, 1023-1056 (1913).

${ }^{2}$ V. Twersky, "Multiple Scattering of Radiation by an Arbitrary Configuration of Parallel Cylinders", The Journal of the Acoustical Society of America 24, 42-46 (1952).

${ }^{3} \mathrm{~S}$. Bose and A. Mal, "Longitudinal shear waves in a fiberreinforced composite", International Journal of Solids and Structures 9, 1075-1085 (1973).

${ }^{4}$ M. Lax, "Multiple Scattering of Waves. II. The Effective Field in Dense Systems", Physical Review 85, 621-629 (1952).

${ }^{5}$ V. K. Varadan, V. V. Varadan, and Y. Pao, "Multiple scattering of elastic waves by cylinders of arbitrary cross section. I. SH waves", The Journal of the Acoustical Society of America 63, 1310-1319 (1978).

${ }^{6}$ P.-Y. Le Bas, F. Luppé, J.-M. Conoir, and H. Franklin, "N-shell cluster in water: Multiple scattering and splitting of resonances", The Journal of the Acoustical Society of America 115, 1460-1467 (2004).

${ }^{7}$ C. M. Linton and P. A. Martin, "Multiple scattering by random configurations of circular cylinders: Second-order corrections for the effective wavenumber", The Journal of the Acoustical Society of America 117, 3413-3423 (2005).
${ }^{8}$ A. N. Norris and J.-M. Conoir, "Multiple scattering by cylinders immersed in fluid: High order approximations for the effective wavenumbers", The Journal of the Acoustical Society of America 129, 104-113 (2011).

${ }^{9}$ G. O. Olaofe, "Scattering by Two Cylinders", Radio Science 5, 1351-1360 (1970).

${ }^{10} \mathrm{~J} . \mathrm{W}$. Young and J. C. Bertrand, "Multiple scattering by two cylinders", The Journal of the Acoustical Society of America 58, 1190-1195 (1975).

${ }^{11}$ R. P. Radlinski and T. J. Meyers, "Radiation patterns and radiation impedances of a pulsating cylinder surrounded by a circular cage of parallel cylindrical rods", The Journal of the Acoustical Society of America 56, 842-848 (1974).

${ }^{12}$ F. A. Amirkulova and A. N. Norris, "Acoustic multiple scattering using recursive algorithms", Journal of Computational Physics 299, 787-803 (2015).

${ }^{13}$ L. Greengard and V. Rokhlin, "A Fast Algorithm for Particle Simulations", ,13 (1987).

${ }^{14}$ S. Koc and W. C. Chew, "Calculation of acoustical scattering from a cluster of scatterers", The Journal of the Acoustical Society of America 103, 721-734 (1998).

${ }^{15}$ N. A. Gumerov and R. Duraiswami, "Computation of scattering from clusters of spheres using the fast multipole method", The Journal of the Acoustical Society of America 117, 1744-1761 (2005).

${ }^{16}$ N. A. Gumerov and R. Duraiswami, Fast Multipole Methods for the Helmholtz Equation in Three Dimensions (Elsevier, 2004).

${ }^{17}$ Y. J. Zhang and E. P. Li, "Fast multipole accelerated scattering matrix method for multiple scattering of a large number of cylinders", Progress In Electromagnetics Research 72, 105-126 (2007).

${ }^{18}$ S. Balay, S. Abhyankar, M. F. Adams, J. Brown, P. Brune, K. Buschelman, L. Dalcin, A. Dener, V. Eijkhout, W. D. Gropp, D. Kaushik, M. G. Knepley, D. A. May, L. C. McInnes, R. T. Mills, T. Munson, K. Rupp, P. Sanan, B. F. Smith, S. Zampini, H. Zhang, and H. Zhang, PETSc Web page, http://www.mcs.anl. gov/petsc, 2018.

${ }^{19}$ S. Balay, S. Abhyankar, M. F. Adams, J. Brown, P. Brune, K. Buschelman, L. Dalcin, A. Dener, V. Eijkhout, W. D. Gropp, D. Kaushik, M. G. Knepley, D. A. May, L. C. McInnes, R. T. Mills, T. Munson, K. Rupp, P. Sanan, B. F. Smith, S. Zampini, H. Zhang, and H. Zhang, "PETSc users manual," Technical Report No. ANL-95/11 - Revision 3.10, Argonne National Laboratory (unpublished).

${ }^{20}$ S. Balay, W. D. Gropp, L. C. McInnes, and B. F. Smith, "Efficient management of parallelism in object oriented numerical software libraries," in Modern Software Tools in Scientific Computing, edited by E. Arge, A. M. Bruaset, and H. P. Langtangen (Birkhäuser Press, 1997), pp. 163-202.

${ }^{21}$ P. Martin, Multiple Scattering - Interaction of Time-Harmonic Waves with $N$ Obstacles (Cambridge University Press, 2006).

22 J. J. Faran, "Sound Scattering by Solid Cylinders and Spheres", The Journal of the Acoustical Society of America 23(4), 405-418 (1951).

${ }^{23}$ N. D. Veksler, Resonance Acoustic Spectroscopy (SpringerVerlag, 1993).

${ }^{24}$ X. Antoine, C. Chniti, and K. Ramdani, "On the numerical approximation of high-frequency acoustic multiple scattering problems by circular cylinders", Journal of Computational Physics 227, 1754-1771 (2008).

${ }^{25}$ T. Valier-Brasier and J.-M. Conoir, "Resonant acoustic scattering by two spherical bubbles", The Journal of the Acoustical Society of America 145, 301-311 (2019).

${ }^{26}$ N. Gould and J. Scott, "The State-of-the-Art of Preconditioners for Sparse Linear Least-Squares Problems", ACM Transactions on Mathematical Software 43, 1-35 (2017).

${ }^{27}$ I. S. Duff, "The impact of high-performance computing in the solution of linear systems: trends and problems", Journal of Computational and Applied Mathematics 123, 515-530 (2000). 
${ }^{28}$ S. Biwa, S. Yamamoto, F. Kobayashi, and N. Ohno, "Computational multiple scattering analysis for shear wave propagation in unidirectional composites", International Journal of Solids and Structures 41, 435-457 (2004).

${ }^{29}$ L. L. Foldy, "The Multiple Scattering of Waves. I. General Theory of Isotropic Scattering by Randomly Distributed Scatterers", Physical Review 67, 107-119 (1945).

${ }^{30}$ Y. Pennec, B. Djafari-Rouhani, J. O. Vasseur, A. Khelif, and P. A. Deymier, "Tunable filtering and demultiplexing in phononic crystals with hollow cylinders", Physical Review E 69, 046608 (2004).

${ }^{31}$ M. Chekroun, L. L. Marrec, B. Lombard, and J. Piraux, "Multiple scattering of elastic waves: a numerical method for computing the effective wavenumbers", Waves in Random and Complex Media 22, 398-422 (2012).

${ }^{32}$ B. Tripathi, A. Luca, S. Baskar, F. Coulouvrat, and R. Marchiano, "Threshold of oscillation of a vocal fold replica with unilateral surface growths", The Journal of the Acoustical Society of America, 298-319 (2017), (published online).

${ }^{33}$ A. Luca, R. Marchiano, and J.-C. Chassaing, "Element centered smooth artificial viscosity in discontinuous galerkin method for propagation of acoustic shock waves on unstructured meshes",
Journal of Computational Physics 63, 886-897 (2018).

${ }^{34} \mathrm{C}$. Aristégui and Y. C. Angel, "Effective mass density and stiffness derived from p-wave multiple scattering", Wave Motion 44, 153-164 (2007).

${ }^{35}$ M.R. Haberman and A.Norris, "Acoustic metamaterials", Acoustics Today 12, 31-39 (2016).

${ }^{36}$ A. Aubry, A. Derode, P. Roux, and A. Tourin, "Coherent backscattering and far-field beamforming in acoustics", The Journal of the Acoustical Society of America 121, 70-77 (2007).

${ }^{37}$ A. Derode, V. Mamou, and A. Tourin, "Influence of correlations between scatterers on the attenuation of the coherent wave in a random medium", Physical Review E 74, 036606 (2006).

${ }^{38}$ D. Torrent and J. Sánchez-Dehesa, "Sound scattering by anisotropic metafluids based on two-dimensional sonic crystals", Physical Review B 79, 174104 (2009).

${ }^{39}$ E. Reyes-Ayona, D. Torrent, and J. Sánchez-Dehesa, "Homogenization theory for periodic distributions of elastic cylinders embedded in a viscous fluid", The Journal of the Acoustical Society of America 132, 2896-2908 (2012).

${ }^{40}$ D. Torrent and J. Sánchez-Dehesa, "Effective parameters of clusters of cylinders embedded in a nonviscous fluid or gas", Physical Review B 74, 224305 (2006). 